\title{
Normal Stress Distribution of Rough Surfaces in Contact
}

\author{
Alex Hansen, ${ }^{1,2}$ Jean Schmittbuhl, ${ }^{3}$ G. George Batrouni, ${ }^{4}$ and Fernando A. \\ de Oliveira \\ International Center for Condensed Matter Physics, Universidade de Brasília, Brazil
}

\begin{abstract}
We study numerically the stress distribution on the interface between two thick elastic media bounded by interfaces that include spatially correlated asperities. The interface roughness is described using the self-affine topography that is observed over a very wide range of scales from fractures to faults. We analyse the correlation properties of the normal stress distribution when the rough surfaces have been brought into full contact. The self affinity of the rough surfaces is described by a Hurst exponent $H$. We find that the normal stress field is also self affine, but with a Hurst exponent $H-1$. Fluctations of the normal stress are shown to be important, especially at local scales with anti-persistent correlations.
\end{abstract}

Theories describing the elastic properties of two media in contact through rough surfaces have important applications in a wide range of geophysical problems, such as earthquakes, fracture, fluid permeability or rock friction [Scholz, 1990]. Asperities exist at all scales: grain roughness is relevant for closure of rock joints [Brown and Scholz, 1986] and seamounts might induce large scale stress fluctuations along subduction slabs [Dmowska et al., 1996]. Whatever the scale of the asperities in contact is, when they are attached to an elastic medium and are loaded, they interact and concentrate high stresses. Friction properties of an interface are very dependent on the heterogeneities of the normal stresses [Dieterich and Kilgore, 1996]. At fault scale, residual stresses resulting from asperity squeeze might be responsible for heterogeneities of the dynamic stress field and influence the earthquake propagation [Bouchon et al., 1998].

We consider in this letter the normal component of the stress field $\sigma_{N}$ that appears on the interface between two elastic media with rough surfaces (see Figure 1) [Sayles, 1996]. We assume that possible local plastic

\footnotetext{
${ }^{1}$ Present address: NORDITA, Blegdamsvej 17, DK-2100 Copenhagen, Denmark

${ }^{2}$ Permanent address: Department of Physics, Norwegian University of Science and Technology, N-7491 Trondheim, Norway

${ }^{3}$ Permanent address: Laboratoire de Géologie, UMR CNRS 8538, Ecole Normale Supérieure, 24, rue Lhomond, F-75231 Paris Cédex 05, France

${ }^{4}$ Permanent address: Institut Non-Linéaire de Nice, UMR CNRS 6618, Université de Nice-Sophia Antipolis, 1361 Route des Lucioles, F-06560 Valbonne, France
}

Figure 1. 
deformations where the elastic yield stress of asperities is overcome, have a negligible effect on the stress distribution along the interface. The roughness is assumed to be self affine. The surface is described as $h(x, y)$. Rescaling the two coordinates $x \rightarrow \lambda x$ and $y \rightarrow \lambda y$, necessitates a rescaling of the height $h \rightarrow \lambda^{H}[$ Feder, 1988]. The surface is then self affine with a Hurst exponent $H$. In Figure 1 an example of surfaces with this property is shown.

This surface was obtained from an impact fracturing of a granite block $(25 \mathrm{~cm} \times 25 \mathrm{~cm})$ [Lòpez and Schmittbuhl, 1998]. A wide range of experimental data [Brown and Scholz, 1985a; Power et al., 1987; Bouchaud et al., 1990; Måløy et al., 1992; Schmittbuhl et al., 1995] support the hypothesis that not only are surfaces produced by brittle fracture self affine, but their Hurst exponent generally equals $H=0.80$ independently of the material [Bouchaud, 1997]. We have measured the roughness of the granite block using a profilometer and analysed the spatial correlations of the surface with the average wavelet coefficient technique [Mehrabi et al., 1997; Simonsen et al., 1998]. This consists in wavelet transforming each one-dimensional trace $h(x, y=$ const) using the Daubechie-12 wavelet basis and averaging over the wavelet coefficients at each length scale $b=2^{k}$, where $k$ is an integer. If the trace is self affine, the averaged wavelet coefficients scale as

$$
A_{b} \sim b^{H+1 / 2}
$$

where $A_{b}$ is an average over the position of the wavelet.

We show in Figure 2 the average wavelet coefficients $v s$. $b$ for the granite surface of Figure 1 . The slope of the least-squares fit is $1.30 \pm 0.02$, giving $H=0.80 \pm 0.02$.

Integrating the Lamé equations for an infinite block Figure 2. limited by an infinite plane $(x, y, z=0)$, gives the Green function $G$ for the deformational response $u$ in the $z$ (vertical) direction at a point $\left(x_{u}, y_{u}\right)$ in the plane $z=0$ from a distribution $f\left(x_{f}, y_{f}\right)$ of applied forces in the vertical direction:

$u\left(x_{u}, y_{u}\right)=\iint G\left(x_{u}-x_{f}, y_{u}-y_{f}\right) f\left(x_{f}, y_{f}\right) d x_{f} d y_{f}$

where [Landau and Lifshitz, 1958]

$$
G\left(x_{u}-x_{f}, y_{u}-y_{f}\right)=\frac{1-s^{2}}{\pi E} \frac{1}{r} .
$$

Here $r=\sqrt{\left(x_{u}-x_{f}\right)^{2}+\left(y_{u}-y_{f}\right)^{2}}, E$ is the elastic constant and $s$ is the Poisson ratio. Deformation also occurs within the $(x, y)$-plane when vertical force is applied. However, these fall of as $1 / r^{2}$. Consequently, we ignore them compared to the deformation in the vertical direction.

We are interested in rough self-affine surfaces. However, with a Hurst exponent $H<1$, the surfaces are asymptotically flat. This can be easily seen by calculating the rms fluctuations of the surface, $w^{2}=\langle(h-$ 
$\left.\langle h\rangle)^{2}\right\rangle_{L \times L}$, where $\langle\cdots\rangle_{L \times L}$ is an average over an area of size $L \times L$ of the plane $(x, y)$. When the surface is self affine, we have $w \sim L^{H}$ leading to $w / L \sim 1 / L^{1-H} \rightarrow 0$ as $L \rightarrow \infty$. Thus, it is asymptotically flat. As we are primarily interested in the scaling properties of the normal component of the stress field $\sigma_{N}$ on large scales, it is a good approximation to use the flat-surface Green function, (3). This approximation also leads to the force component in the vertical direction being directly proportional to the normal stress.

The problem we have set out to study is that of two self-affine rough surfaces in full contact. However, assuming that one of the surfaces is rough and infinitely hard, and the other elastic and initially flat, we find the same normal stress field at the interface as in the original problem within the approximation using the flat-surface Green function, (3) and using the composite topography introduced by Brown and Scholz [1985b] (i.e. the sum of both topographies). We will, therefore, study this second problem since it is easier to implement numerically.

When the two surfaces are in full contact, the deformation field $u$ will be equal to minus the local height, $u=-h$, when in-plane deformations are ignored. Thus, the deformation field is self-affine, with a Hurst exponent $H$.

Since Eq. (2) is linear, simple scaling arguments tell us how the force field $f$ scales given that $u$ is self affine. ${ }^{1}$ If we scale $\left(x_{u}, y_{u}\right) \rightarrow\left(\lambda x_{u}, \lambda y_{u}\right)$ and $\left(x_{f}, y_{f}\right) \rightarrow\left(\lambda x_{f}, \lambda y_{f}\right)$, Eqs. (2) and (3) immediately gives the scaling relations

$$
\left\{\begin{array}{l}
\Delta x \rightarrow \lambda \Delta x, \\
\Delta y \rightarrow \lambda \Delta y, \\
u \rightarrow \lambda^{H} u \\
G \rightarrow \lambda^{-1} G, \\
f \rightarrow \lambda^{H-1} f,
\end{array}\right.
$$

with $\Delta x=x_{u}-x_{f}$ and $\Delta y=y_{u}-y_{f}$. Thus, the force field $f$ is self affine with a Hurst exponent equal to $H_{\sigma}=H-1$.

In order to demonstrate the validity of Eq. (4), we solve Eq. (2) numerically for $f$. This is done in Fourier space (using FFTs [Stanley and Kato, 1997]) since the Green function is diagonal there. We start out by defining the Green function on the $L \times L$ square lattice as follows. For each node $(i, j)$, we define $r_{1}=$ $\left((i-1)^{2}+(j-1)^{2}\right)^{1 / 2}, r_{2}=\left((i-1)^{2}+(L+1-j)^{2}\right)^{1 / 2}$, $r_{3}=\left((L+1-i)^{2}+(j-1)^{2}\right)^{1 / 2}$, and $r_{4}=\left((L+1-i)^{2}+\right.$ $\left.(L+1-j)^{2}\right)^{1 / 2}$. The Green function on this lattice is then given by

$$
G_{(i, j)}=\frac{1-s^{2}}{4 \pi E}\left[\frac{1}{\max \left(r_{1}, \epsilon\right)}+\frac{1}{r_{2}}+\frac{1}{r_{3}}+\frac{1}{r_{4}}\right] .
$$

\footnotetext{
${ }^{1}$ If the underlying equations were not linear, much more powerful methods to determine the scaling behavior would be necessary such as functional renormalization [Barabási and Stanley, 1997].
} 
Thus, the singularity of the Green function is situated at $(i=1, j=1)$. We have introduced a cutoff in $r_{1}$ equal to $\epsilon$. We choose it to be a quarter of the lattice spacing, i.e., 1/4. The reason for introducing the three other radii $r_{2}, r_{3}$, and $r_{4}$ is that the Fourier transform makes the lattice periodic. The three additional radii signify the mirror image of the singularity resulting from one reflection - we do not introduce further reflections since, with our choice of parameters, their effect is negligible. The deformation field $u$ and the Green fuction, $G$ were then Fourier transformed, and Eq. (2) solved in Fourier space. The resulting force field was then Fourier transformed back to real space. To within the approximations we have made, the force field is proportional to the normal stress field $\sigma_{N}$. We show in Figure 3 the normal stress field corresponding to the full contact of the fracture in Figure 1. The strong small-scale variations in the normal stress distribution are consistent with the observations reported in Mendelsohn et al. [1998].

In Figure 2, we show the wavelet analysis of the normal stress field obtained for the granite surface. The least-squares fit gives a slope of $0.30 \pm 0.04$ corresponding to a Hurst exponent of $-0.20 \pm 0.04$. Thus, the relation $H_{\sigma}=H-1$ is supported.

In order to study systematically the relation between the Hurst exponent of the deformation field and that of the normal stress field, we have generated artificial selfaffine surfaces, using the Fourier method [Sahimi, 1998]. This allows us to generate and subsequently average our results over many surfaces for each Hurst exponent, in practice 100 surfaces. After obtaining the stress fields for each surface, we analysed spatial correlations of both the surfaces and the stress fields with two techniques: the average wavelet spectrum of one-dimensional traces obtained by cutting the surface along lines and the two dimensional Fourier spectrum of the full surface. Figure 2 presents the average wavelet spectra of the surfaces and their corresponding stress field for the synthetic fracture surfaces with the same Hurst exponent as the one that is observed for fracture surfaces: $H=0.80$. We treated each surface and its corresponding stress field as consisting of 2048 one-dimensional traces, and as there were 100 surfaces, our averages are over $100 \times 2048$ one-dimenensional traces. The scaling of the synthetic surfaces is in good agreement with that of the measured surface. Computed full contact stress fields of both types of surfaces are also in good agreement supporting the relation: $H_{\sigma}=H-1$. The two dimensional Fourier spectrum is computed from the two dimensional Fourier transform of the surface and is expected to scale for self-affine surfaces as [Sahimi, 1998]:

$$
P(|\mathbf{k}|) \sim|\mathbf{k}|^{-2-2 H}
$$

We generalize the analysis for different Hurst exponents that describe different spatial correlations between asperities. In Figure 4, we show $H_{\sigma}$ as a function of $H$ for the artificially generated $2048 \times 2048$ surfaces 
and analyses with both techniques. The straight line corresponds to

$$
H_{\sigma}=H-1 .
$$

We see that the numerical results and Eq. (7) are in excellent agreement for the two dimensional Fourier spectrum. The agreement is good with the one dimensional technique only for sufficiently large roughness exponents of the rough surface. We emphasize that measurements of low roughness exponent have to be done with two dimensional techniques [Hansen et al., 2000].

The Hurst exponent is directly related to the spatial correlations of the surface. Eq. 6 shows that for: $H=-1$ surfaces have a flat spectrum that is are white noise with no spatial correlations of the asperities. When the Hurst exponent $H$ increases, relative magnitudes of low frequency modes also increase. Asperities are smoother and the surface roughness appears more and more correlated at large scales. Equation (7) shows that the stress field can be calculated approximately as a simple derivative of the deformation field. Also, fluctations of the stress field are significantly higher than the deformation field.

We thank H. Nazareno of the ICCMP for the opportunity to perform this work in an excellent and friendly environment. This work was partially funded by the CNRS PICS contract \#753 and the Norwegian research council, NFR. We also thank NORDITA for its hospitality.

\section{References}

Barabási, A., and H. Stanley, Introduction to Fractal Surface Growth. Cambridge University Press, Cambridge, 1997.

Bouchaud, E., Scaling properties of cracks, J. Phys.: Condens. Matter, 9, 4319-4344, 1997.

Bouchaud, E., G. Lapasset and J. Planès, Fractal dimension of fractured surfaces: a universal value? Europhys. Lett. 13, 73-79, 1990.

Bouchon, M., M. Campillo, and F. Cotton, Stress field associated with the rupture of the 1992 Landers, California, earthquake and its implications concerning the fault strength at the onset of the earthquake, J. Geophys. Res., 103, 21091-21097, 1998.

Brown, S. R., and C. H. Scholz, Broad bandwidth study of the topography of natural rock surfaces, J. Geophys. Res., 90, 12575-12582, 1985a.

Brown, S. R., and C. H. Scholz, Closure of random elastic surfaces in contact, J. Geophys. Res., 90, 5531-5545, 1985b.

Brown, S. R., and C. H. Scholz, Closure of Rock Joints, J. Geophys. Res., 91, 4939-4948, 1986.

Dieterich, J., and B. Kilgore, Imaging surface contacts: Power law contact distributions and contact stresses in quartz, calcite, glass and acrylic plastic, Tectonophysics, 256, 216-239, 1996.

Dmowska, R., R. Zheng, and J. Rice, Seismicity and deformation at convergent margins due to heterogeneous coupling, J. Geophys. Res., 101, 3015-3029, 1996.

Feder, J., Fractals. Plenum, New York, 1988.

Hansen, Alex, J. Schmittbuhl, and G.G. Batrouni, Distinguishing Fractional and White Noise in One and Two Dimensions, in preparation, 2000. 
Landau, L., and E. Lifchitz, Theory of elasticity. Pergamon Press, Oxford, 1958.

Lòpez, J., and J. Schmittbuhl, Anomalous scaling of fracture surfaces, Phys. Rev. E, 57, 6405-6408, 1998.

Måløy, K. J., A. Hansen, E. L. Hinrichsen and S. Roux, Experimental measurements of the roughness of brittle cracks, Phys. Rev. Lett. 68, 213-215, 1992.

Mehrabi, A. R., H. Rassamdana and M. Sahimi, Characterization of long-range correlations in complex distributions and profiles, Phys. Rev. E, 56, 712-722, 1997.

Mendelsohn, D. A., T. S. Gross, R. U. Goulet and M. Zhouc, Experimental-computational estimation of rough fracture surface contact stresses, Materials Sci. and Eng. A249, 16, 1998.

Power, W. L., T. E. Tullis, S. R. Brown, G. N. Boitnott, and C. H. Scholz, Roughness of natural fault surfaces, Geophys. Res. Lett., 14, 29-32, 1987.

Sahimi, M., Non-linear and non-local transport process in heterogeneous media: from long-range correlated percolation to fracture and material breakdown, Phys. Reports, 306, 213-395, 1998.

Sayles, R. S., Basic principles of rough surface contact analysis using numerical methods, Tribol. Int. 29, 639-650, 1996.

Schmittbuhl, J., F. Schmitt, and C. H. Scholz, Scaling invariance of crack surfaces, J. Geophys. Res., 100, 59535973, 1995.

Scholz, C. H., The mechanics of Earthquakes and faulting. Cambridge Univ. Press, New York, 1990.

Simonsen, I., A. Hansen, and O. M. Nes, Using wavelet transforms for Hurst exponent determination, Phys. Rev. E, 58, 2779, 1998.

Stanley, H. M., and T. Kato, An FFT-based method for rough surface contact, ASME Journal of Tribology, 119,481-485, 1997.

G. George Batrouni, Institut Non-Linéaire de Nice, UMR CNRS 6618, Université de Nice-Sophia Antipolis, 1361

Route des Lucioles, F-06560 Valbonne, France

Alex Hansen, Department of Physics, Norwegian University of Science and Technology, N-7491 Trondheim, Norway

Fernando A. de Oliveira, ICCMP, Universidade de Brasília, CP 04513, 70919-970 Brasília-DF, Brazil

Jean Schmittbuhl, Laboratoire de Géologie, UMR CNRS

8538, Ecole Normale Supérieure, 24, rue Lhomond, F-75231

Paris Cédex 05, France

(Received March xx, 2000) 
HANSEN ET AL: STRESS OF ROUGH SURFACES

HANSEN ET AL: STRESS OF ROUGH SURFACES

HANSEN ET AL: STRESS OF ROUGH SURFACES

HANSEN ET AL: STRESS OF ROUGH SURFACES

HANSEN ET AL: STRESS OF ROUGH SURFACES

HANSEN ET AL: STRESS OF ROUGH SURFACES

HANSEN ET AL: STRESS OF ROUGH SURFACES

HANSEN ET AL: STRESS OF ROUGH SURFACES

HANSEN ET AL: STRESS OF ROUGH SURFACES

HANSEN ET AL: STRESS OF ROUGH SURFACES

HANSEN ET AL: STRESS OF ROUGH SURFACES

HANSEN ET AL: STRESS OF ROUGH SURFACES

HANSEN ET AL: STRESS OF ROUGH SURFACES

HANSEN ET AL: STRESS OF ROUGH SURFACES 


\section{Figure Captions}

Figure 1. A rough granite surface produced by cleavage. The size is $512 \times 512$ points. Physical dimensions are $12.8 \mathrm{~mm} \times 12.8 \mathrm{~mm}$.

Figure 2. The average wavelet coefficient $A_{b}$ for the fracture surface of Figure 1 as a function of length scale $b$ is shown as filled circles. Empty circles correpond to the average over 100 synthetic surfaces of $2048 \times 2048$. The slope of the straight solid line is 1.30 , corresponding to a Hurst exponent $H=0.80$. Average wavelet coefficient $A_{b}$ for the stress field shown in Figure 3 as a function of length scale $b$ using filled diamonds. Empty diamonds describe the analysis of the squeeze of the synthetic surfaces. The slope of the dashed straight line is 0.30 , corresponding to a Hurst exponent $H_{\sigma}=-0.2$.

Figure 3. The numerically calculated stress field on the interface between the granite block of Figure 1 when brought in complete contact with an infinitely hard flat surface.

Figure 4. Hurst exponent of stress field, $H_{\sigma}$ as function of Hurst exponent of deformation field $H$. The data are based on averaging over several samples of artifially generated rough surfaces $(\diamond$ for $1 \mathrm{D}$ wavelet spectrum and $\circ$ for $2 \mathrm{D}$ Fourier spectrum). The straight line is $H_{\sigma}=H-1$. 
Figures

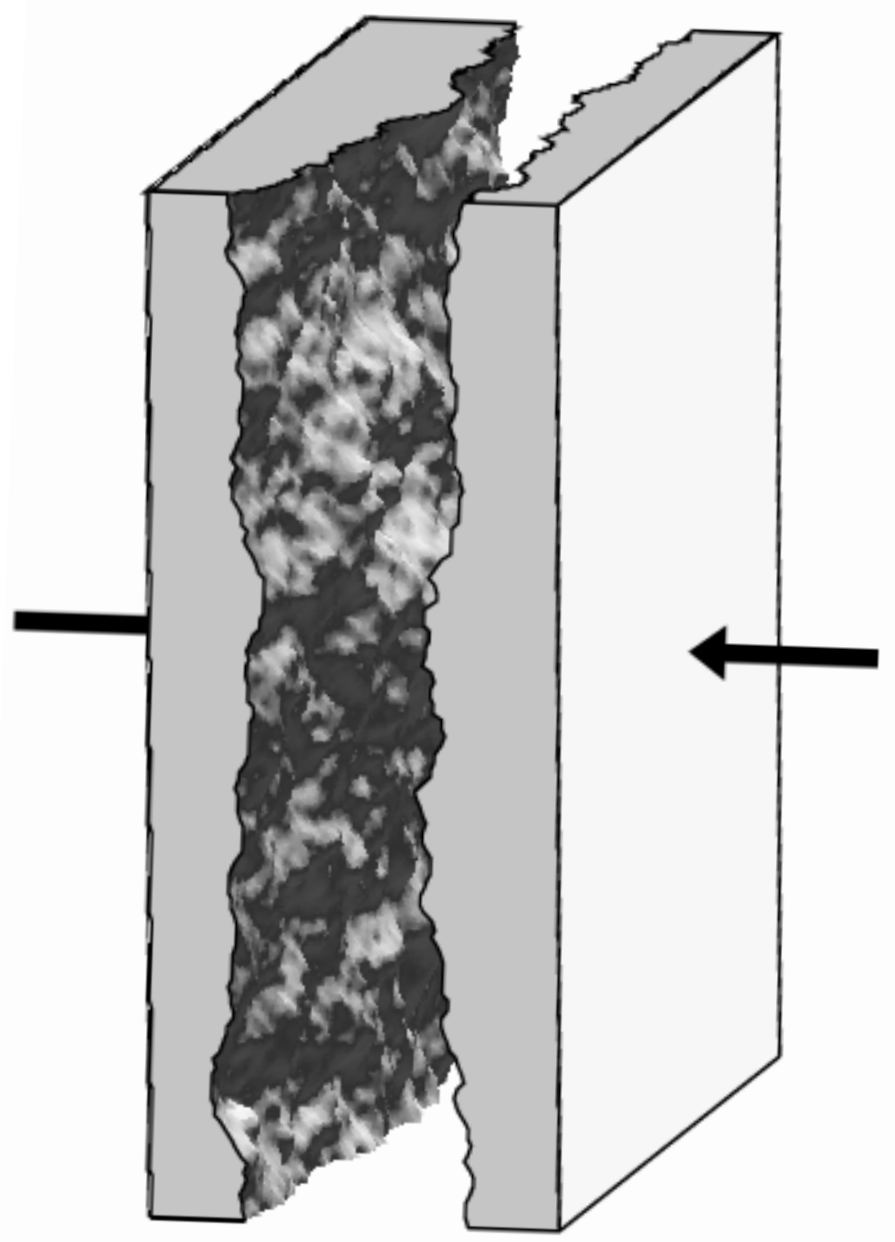

Figure 1. A rough granite surface produced by cleavage. The size is $512 \times 512$ points. Physical dimensions are $12.8 \mathrm{~mm} \times 12.8 \mathrm{~mm}$. 


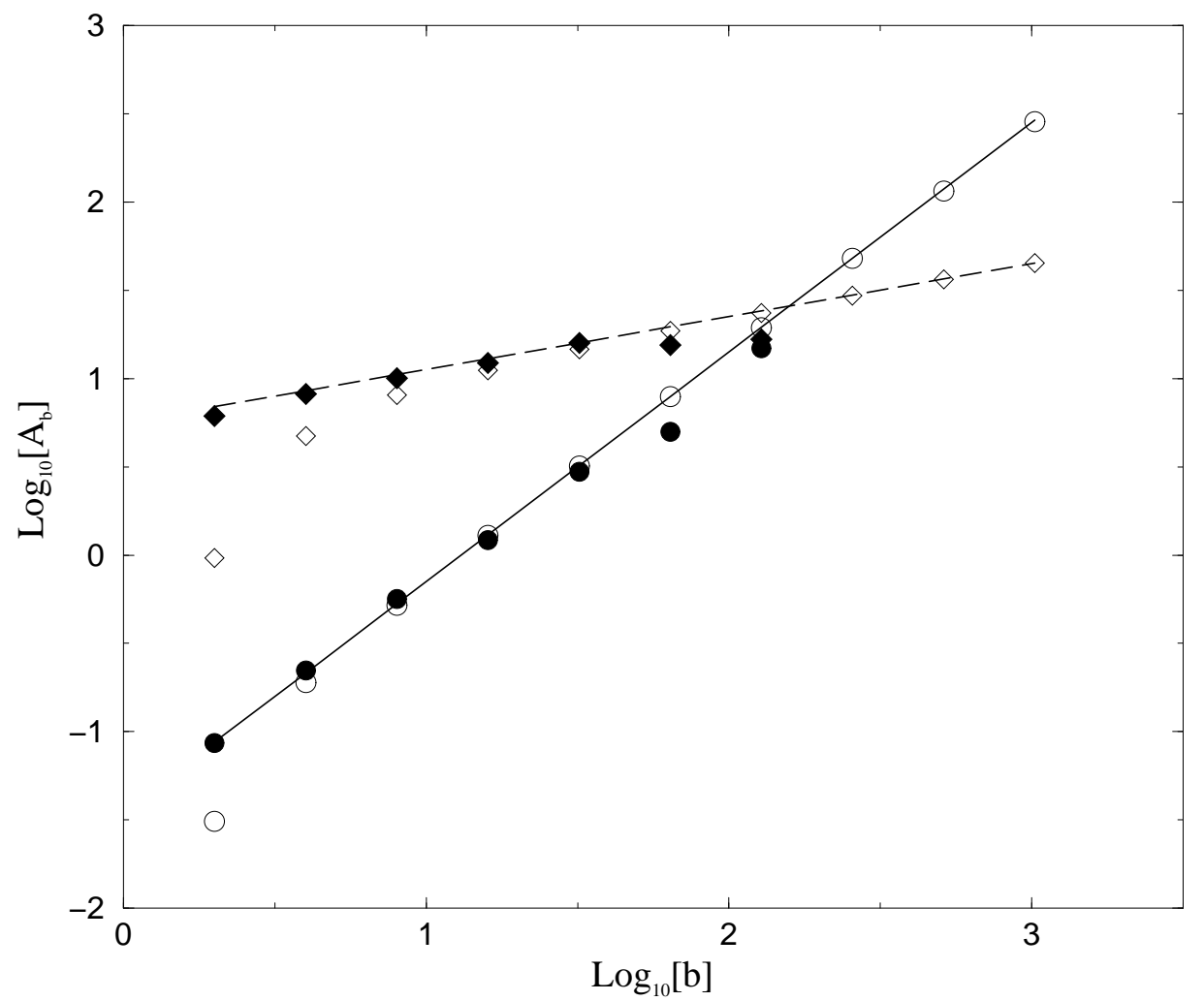

Figure 2. The average wavelet coefficient $A_{b}$ for the fracture surface of Figure 1 as a function of length scale $b$ is shown as filled circles. Empty circles correpond to the average over 100 synthetic surfaces of $2048 \times 2048$. The slope of the straight solid line is 1.30 , corresponding to a Hurst exponent $H=0.80$. Average wavelet coefficient $A_{b}$ for the stress field shown in Figure 3 as a function of length scale $b$ using filled diamonds. Empty diamonds describe the analysis of the squeeze of the synthetic surfaces. The slope of the dashed straight line is 0.30 , corresponding to a Hurst exponent $H_{\sigma}=-0.2$. 


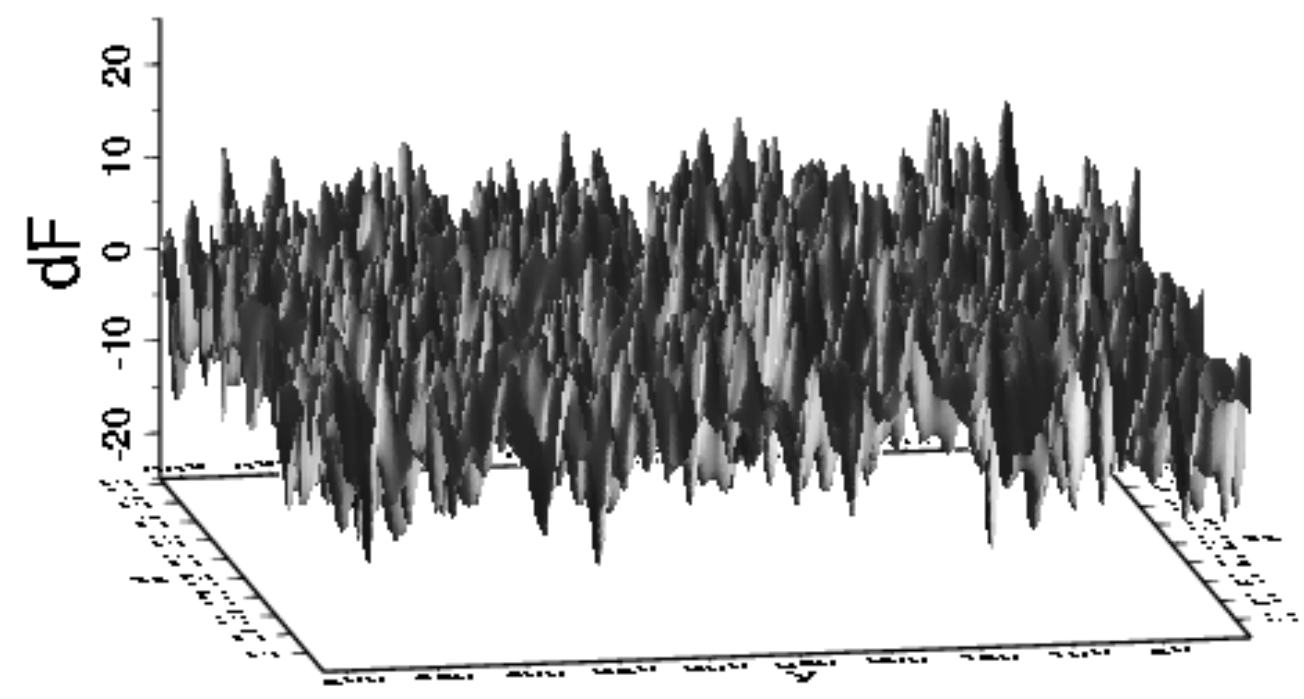

Figure 3. The numerically calculated stress field on the interface between the granite block of Figure 1 when brought in complete contact with an infinitely hard flat surface.

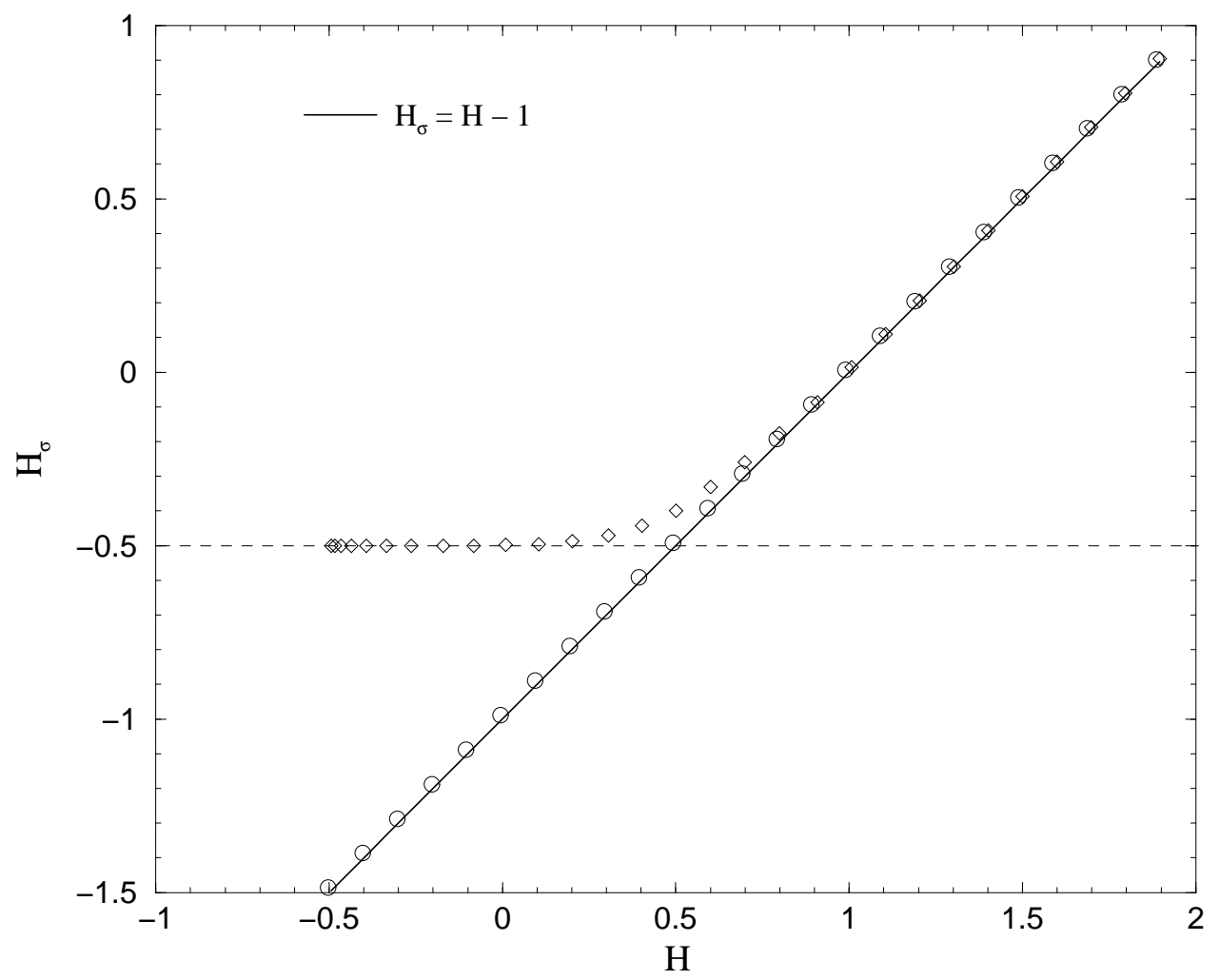

Figure 4. Hurst exponent of stress field, $H_{\sigma}$ as function of Hurst exponent of deformation field $H$. The data are based on averaging over several samples of artifially generated rough surfaces ( $\diamond$ for $1 \mathrm{D}$ wavelet spectrum and $\circ$ for $2 \mathrm{D}$ Fourier spectrum). The straight line is $H_{\sigma}=H-1$. 